\title{
Fano resonance in the luminescence spectra of donor bound excitons in polar semiconductors
}

\author{
Kui-juan $\operatorname{Jin}^{\mathrm{a})}$ \\ Department of Physics, The University of Hong Kong, Pokfulam Road, Hong Kong, China; HKU-CAS Joint \\ Laboratory on New Materials, The University of Hong Kong, Pokfulam Road, Hong Kong, China; \\ and Institute of Physics, Chinese Academy of Sciences, P.O. Box 603, Beijing 100080, China \\ S. J. Xu \\ Department of Physics, The University of Hong Kong, Pokfulam Road, Hong Kong, China \\ and HKU-CAS Joint Laboratory on New Materials, The University of Hong Kong, Pokfulam Road, \\ Hong Kong, China
}

(Received 17 October 2006; accepted 14 December 2006; published online 17 January 2007)

\begin{abstract}
An unusual response in the luminescence measurements of $\mathrm{GaN}$ thin film and $\mathrm{ZnO}$ bulk crystal is observed at low temperatures. The authors demonstrate theoretically that such an unusual response is due to the longitudinal optical phonon mediated Fano resonance involved in the recombination process of the donor bound exciton. The line shapes obtained by the calculation in the present mechanism are in excellent agreement with the experimental results. (C) 2007 American Institute of Physics. [DOI: 10.1063/1.2432286]
\end{abstract}

The quantum-mechanical interference between a discrete state and a continuum of states is a fundamental problem in physics that was first treated by Fano ${ }^{1}$ and Anderson ${ }^{2}$ independently. The quantum interference manifests itself as a resonance in the spectrum with a characteristic asymmetric line shape. It has been observed in a large number of very different physical systems, such as atomic, molecular, ${ }^{3}$ and semiconducting $^{4-9}$ systems. These studies on the Fano effect are mainly concerned with the photon absorption type transitions such as the photoconductive process ${ }^{10}$ or light inelastic scattering processes such as Raman scattering. On the other hand, in another fundamental type of optical process, i.e., photon emission, relevant studies on the Fano resonance are relatively few. ${ }^{11}$ In particular, the experimental observation of the Fano resonance in the photon emission of excitons in solids is very few. Very recently, we have observed the Fano resonance line shape of the first-order longitudinal optical (LO) phonon-assisted luminescence of the donor bound excitons in high quality $\mathrm{GaN}$ epilayers and $\mathrm{ZnO}$ single crystals. ${ }^{12,13}$ A firm identification of such an unusual line shape in the exciton luminescence spectra certainly requires a theoretical confirmation.

In this letter, we theoretically show that the unusual features with asymmetric line shapes detected in the first-order LO phonon sidebands of the neutral-donor-bound excitons of the semiconductors originate from the Fano-type interference caused by the coupling between the weakly dispersed LO phonon state and a continuum of exciton-acoustic-phonon coupling states ${ }^{14,15}$ in the materials, where the exciton vacuum state serves as the common final state of Fano resonance.

The high quality GaN sample used in the measurements was a $2.88 \mu \mathrm{m} \mathrm{GaN}$ layer grown followed by a $40 \mathrm{~nm} \mathrm{GaN}$ nucleation layer. The $\mathrm{ZnO}$ sample was a high quality bulk crystal. In the photoluminescence (PL) measurements, the samples were mounted on the cold finger of a Janis closedcycle cryostat with varying temperature from 3.5 to $300 \mathrm{~K}$

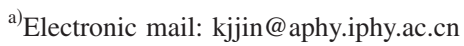

and excited by the $325 \mathrm{~nm}$ line of a Kimmon $\mathrm{He}-\mathrm{Cd} \mathrm{cw}$ laser with an output power of $40 \mathrm{~mW}$. The emission signal was dispersed by a SPEX 750M monochromator and detected with a Hamamatsu R928 photomultiplier.

It has been well established that excitonic radiative transition plays a fundamental role in the PL processes in high quality $\mathrm{GaN}$ and $\mathrm{ZnO}$. Besides the intense exciton resonance luminescence lines, the LO-phonon-assisted luminescence lines of excitons in $\mathrm{GaN}$ and $\mathrm{ZnO}$ are also observed at $3.5 \mathrm{~K}$, as shown in Fig. 1 by squares. The first-order LO phonon replica $\left(D^{0}, X-1 \mathrm{LO}\right)$ of the $D^{0}, X$ line (neutral-donor-bound exciton) dominates in the measured spectral range. An excitonic transition (LX) may be from the radiative recombination of the excitons localized at extended defects in the GaN film. This peak basically quenches when temperature is above $50 \mathrm{~K},{ }^{12}$ indicating its small localization energy. The first-order LO phonon replica (FXA-1LO) of free exciton transition in $\mathrm{GaN}$ (Ref. 12) and the two electron satellite (TES) in ZnO (Ref. 16) can also be seen in Figs. 1(a) and 1(b), respectively. Our variable-temperature PL experiments (not shown here) show that the TES first increases with increasing temperature in the temperature range from 5 to $20 \mathrm{~K}$, and then decreases until it becomes unresolvable. In Fig. 1(b), the peak denoted by $\mathrm{ZPL}_{2}$ is likely the zero phonon line of a deeper bound exciton relative to the $D^{0}, X$, since its phonon replicas can be well interpreted by the Huang-Rhys theory ${ }^{17}$ based on adiabatic approximation. It is worth mentioning that nonadiabatic treatment of electron (exciton)-phonon coupling has been recently addressed. ${ }^{18}$ While the $\mathrm{ZPL}_{1}$ (the zero phonon line of the neutral-donorbound exciton) has been well identified as $D^{0}, X$, the physical nature of the $\mathrm{ZPL}_{2}$ has not been verified to date. Here we thus temporarily denote it as $\mathrm{ZPL}_{2}$.

In the photoluminescence spectra of $\mathrm{GaN}$ and $\mathrm{ZnO}$, the phonon-assisted transition features were revealed. In particular, an abnormal feature with a distinct dip-peak structure around the $D^{0}, X-1 \mathrm{LO}$ lines is observed. This asymmetric feature occurs at the energy of $3.397 \mathrm{eV}$ for $\mathrm{GaN}$ and $3.289 \mathrm{eV}$ for $\mathrm{ZnO}$, which is almost one $\mathrm{LO}$ phonon energy 


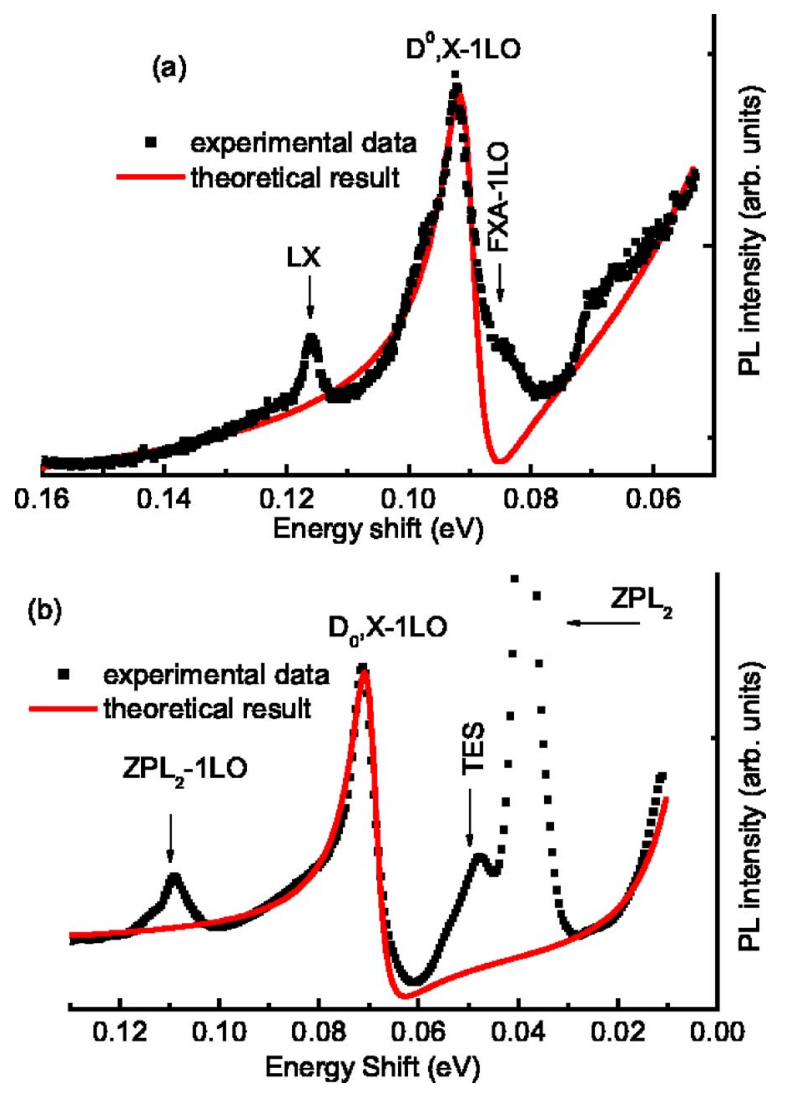

FIG. 1. (Color online) Experimental LO-phonon-assisted PL spectra (solid squares) and corresponding theoretical results (solid lines) based on the Fano resonance process of $\mathrm{GaN}$ (a) and $\mathrm{ZnO}$ (b).

(0.091 eV for $\mathrm{GaN}$ and $0.071 \mathrm{eV}$ for $\mathrm{ZnO}$ ) lower than the energy (3.4896 eV for $\mathrm{GaN}$ and $3.3600 \mathrm{eV}$ for $\mathrm{ZnO}$ ) of the $D^{0}, X$ principal line. Just for simplicity and convenience in comparison with the theoretical results (solid lines), we take the energy zero point at the $D^{0}, X$ line $(3.4896 \mathrm{eV}$ for $\mathrm{GaN}$ and $3.3600 \mathrm{eV}$ for $\mathrm{ZnO}$ ) in Fig. 1. Both different material systems exhibit a similar distinct dip-peak structure around the first-order LO phonon replicas of their neutral-donorbound-exciton lines, which certainly indicate that someone quantum-mechanical interference takes place in the relevant optical transitions. To understand these interesting spectral features, we suggest a reasonable mechanism involving Fano resonance. In general, a Fano resonance occurs when transitions, whatever the excitation mechanism is, ${ }^{1}$ couple the discrete state and continuum to a common ground state and when the discrete state quantum-mechanically interacts with the continuum. It is well known that the line shape with Fano effect features a small energy shift, linewidth broadening, as well as a pronounced intensity minimum near one side of the peak depending on the sign of the Fano asymmetry parameter. From Fig. 1, one can clearly see the asymmetry, the broadening, and the small energy shift towards the lower energy side (i.e., a little bit of departure from the standard $\mathrm{LO}$ phonon energies of $\mathrm{GaN}$ and $\mathrm{ZnO}$ ), in particular, a clear dip at the higher energy side in the line shapes of $D^{0}$, $X-1 \mathrm{LO}$ with respect to those of the corresponding $D^{0}, X$. All these spectral features are exactly consistent with the spectral characteristics of Fano resonance, which makes us to believe that the Fano resonance is observed in the phonon-assisted PL spectra of the bound excitons in polar semiconductors for the first time. As theoretically described below, the phonon-

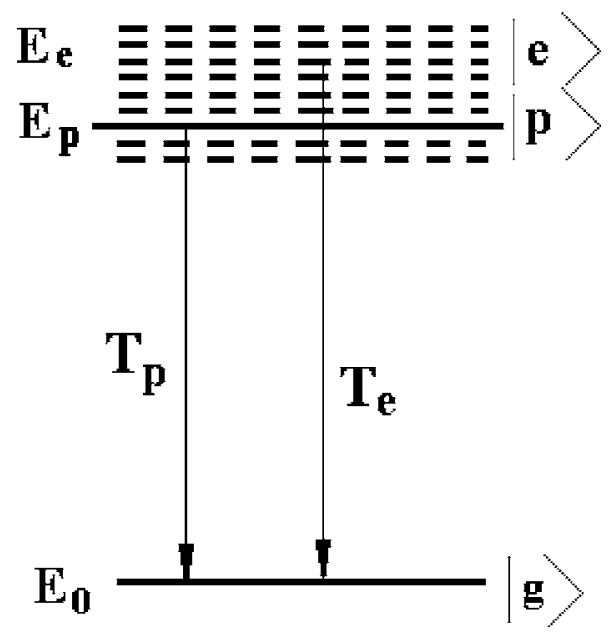

FIG. 2. Schematic illustration of the states and transitions in the system involved in a Fano resonance from the coupling between a LO phonon state and a continuum.

mediated Fano resonance can be attributed to such a physical configuration in which the direct transition from a continuum of exciton-acoustic-phonon coupling states to the excitonic ground state interferes with the indirect transition from an exciton-LO-phonon coupled state to the same ground state of the excitons.

For the systems under study, the excitonic ground state serves as the common final state in the Fano resonance. As the energy of the discrete exciton-LO-phonon coupled state falls within the continuum of exciton-acoustic-phonon coupled states, interaction between the discrete state and the continuum takes place. This leads to a Fano-type interference in the photon emission, which results in the appearance of the PL response with the asymmetric dip-peak line shape. Figure 2 schematically shows the states and the transitions in the systems. Suppose that $T_{e}=\left\langle N, \phi_{0}\left|H_{e l}\left(\hbar \omega_{l}\right)\right| \phi_{e}, N\right\rangle$ is the direct transition matrix element of the electron from excitonacoustic-phonon coupled states $|e\rangle=\left|\phi_{e}, N\right\rangle$ with the energy $E_{e}$ to the ground state $|g\rangle=\left|\phi_{0}, N\right\rangle$ with the energy $E_{0}=0$ and the phonon number $N . \quad T_{p}=\Sigma_{e}\left\langle N, \phi_{0}\left|H_{e l}\left(\hbar \omega_{l}\right)\right| \phi_{e}, N\right\rangle$ $\times\left\langle N, \phi_{e}\left|H_{e p}\right| \phi_{x}, N-1\right\rangle /\left(E_{x}-\hbar \omega_{p}-E_{e}\right)$ is the matrix element of the indirect transition from exciton state $\left|e_{x}\right\rangle=\left|\phi_{x}, N-1\right\rangle$ with the energy $E_{x}$ to the phonon emitted ground state. $V$ $=\left\langle N, \phi_{p}\left|H_{e p}\right| N-1, \phi_{x}\right\rangle$ is the matrix element of electron-LOphonon interaction, where $\left|\phi_{p}, N\right\rangle$ denotes one state among the continuum $\left|\phi_{e}, N\right\rangle$ with the energy of $E_{p}=E_{x}-\hbar \omega_{p}$. Here $\omega_{p}$ is the frequency of LO phonon. $H_{e l}$ and $H_{e p}$ represent the electron-photon and electron-phonon interaction Hamiltonians, respectively. Here $\hbar \omega_{l}$ is the photon energy. This electron-LO-phonon coupling produces a mutual repulsion of the levels $E_{e}$ and $E_{p}$ to positions $E_{ \pm}$, which are the roots of the secular equation $\left(E_{p}-E\right)\left(E_{e}-E\right)-V^{2}=0$. As frequently done to approximate the transition process in other systems, ${ }^{6,710}$ we assume here for simplicity that both $T_{e}$ and $V$ are both constants for each level $|e\rangle$ in the continuum. The line shape of the PL spectrum at LO phonon energy is proportional to $I(E) \propto \sum_{a= \pm}|\langle g|H| a\rangle|^{2} \delta\left(E_{a}-E\right)-|\langle g|H| p\rangle|^{2} \delta\left(E_{p}\right.$ $-E) /|\langle g|H| e\rangle|^{2}$. The first term in the numerator of the righthand fraction of Eq. (1) gives the rate of a direct optical transition, say the transition from a coupled state $\left|E_{a}\right\rangle$ to the ground state. The second term is the rate of indirect transition, in which an electron at an exciton state firstly transits to 
a level with energy of $E_{p}=E_{x}-\hbar \omega_{p}$ within the unperturbed continuum by emitting a LO phonon, and then radiatively decays to the ground state via emitting a photon with the energy of $E_{p}$. Due to the existence of coupled states, an interference between the two kinds of optical transitions occurs. As mentioned earlier, for the direct transition $|e\rangle \rightarrow|g\rangle$, its amplitude is $T_{e}$. For the indirect transition $|e\rangle \rightarrow|p\rangle \rightarrow|g\rangle$, its amplitude can be derived as $V T_{P}\left(E-E_{p}\right)^{-1}$. By employing a similar derivation method used by Fano ${ }^{1}$ and Klein, ${ }^{6}$ Eq. (1) can be further written as $I(E) \propto D(E)(q+\varepsilon)^{2} /\left(1+\varepsilon^{2}\right)$, where $D(E)$ is the density of the continuum states, the reduced energy $\varepsilon$ can be calculated by $\varepsilon=\left[E-E_{p}\right.$ $\left.-V^{2} R\left(E_{p}\right)\right] / \pi V^{2} D\left(E_{p}\right)$, and the Fano asymmetry parameter can be deduced as $q=\left[P \int D(E)\left(E_{p}-E\right)^{-1} d E+i \pi \Sigma_{e} \delta\left(E_{e}\right.\right.$ $\left.\left.-E_{p}\right)+R\left(E_{p}\right)\right] / \pi D\left(E_{p}\right)$. Besides the dip-peak structure caused by the Fano resonance, the continuum states in the semiconductors can produce a continuous PL background which is proportional to the density of states $D(E)$. For simplicity but not losing generality, we take $D(E)$ by an analytical Lorentz shape $D(E)=A\left(1 /\left(E^{2}+B^{2}\right)\right)$, where $E$ denotes the energy in Fig. 1, and $A$ and $B$ are constant parameters for the semiconductors. The Lorentzian parameters are used to fit the tail of $D^{0}, X$, which serves as the background for other PL features. The asymmetry parameter we obtained is 1.12 $+i$ for $\mathrm{GaN}$ and $1.8+i$ for $\mathrm{ZnO}$. Note that the theoretical asymmetry parameter for $\mathrm{ZnO}$ is consistent with the value obtained from the fitting to the experimental spectra using the standard Fano line shape function. ${ }^{13}$ The numerical results are shown in Fig. 1 by solid lines, which are in good agreement with the experimental data, indicating that the phonon-mediated Fano resonance is the physical origin of the observed dip-peak features of the first-order LO phonon replicas.

In summary, the unusual photoluminescence spectra of the bound excitons coupled to $\mathrm{LO}$ phonons in $\mathrm{GaN}$ and $\mathrm{ZnO}$ have been observed at low temperatures. It is shown that Fano resonance due to the coupling between LO phonon and exciton-acoustic-phonon coupling states results not only in the constructive enhancement of the electronic transitions from the exciton-acoustic-phonon coupling states to the ground state but also in the destructive reduction of the electronic transitions, thus leading to the unusual luminescence features with asymmetric dip-peak line shapes around LO phonon energies of the polar semiconductors. The theoretical results obtained in the present mechanism are quantitatively in excellent agreement with the experimental data, which demonstrates that the Fano effect accounts for, in a clear and intuitive way, the nature of the observed dip-peak structure in the LO-phonon-assisted photoluminescence in these polar semiconductor systems.

The work in Hong Kong was supported by the HK-RGC CERG (Grant No. HKU 7056/06P). One of the authors (K.J.J.) gratefully acknowledges research support from the Chinese National Foundation of Nature Sciences. The other author (S.J.X.) wishes to thank S. L. Shi, X. D. Luo, and J. $\mathrm{N}$. Wang for their contributions in the PL measurements of $\mathrm{ZnO}$.

${ }^{1}$ U. Fano, Phys. Rev. 124, 1866 (1961).

${ }^{2}$ P. W. Anderson, Phys. Rev. 124, 41 (1961).

${ }^{3}$ M. Glass-Maujean, H. Frohlich, and J. A. Beswick, Phys. Rev. Lett. 61, 157 (1988).

${ }^{4}$ D. A. Broido and L. J. Sham, Phys. Rev. B 34, 3917 (1986); H. Chu and Y.-C. Chang, ibid. 39, 10861 (1989); K. Maschke, P. Thomas, and E. O. Göbel, Phys. Rev. Lett. 67, 2646 (1991); D. Y. Oberli, G. Böhm, G. Weimann, and J. A. Brum, Phys. Rev. B 49, 5757 (1994).

${ }^{5}$ S. Bar-Ad, P. Kner, M. V. Marquezini, S. Mukamel, and D. S. Chemla, Phys. Rev. Lett. 78, 1363 (1997); D. E. Nikonov, A. Imamoğlu, and M. O. Scully, Phys. Rev. B 59, 12212 (1999); H. Nakata, K. Yamada, and T. Ohyama, Phys. Rev. B 60, 13269 (1999).

${ }^{6}$ M. V. Klein, in Light Scattering in Solid, 2nd ed., Topics in Applied Physics Vol. 8, edited by M. Cardona (Springer, Berlin, 1975), p. 169; M. Cardona, F. Cerdeira, and T. A. Fjeldly, Phys. Rev. B 10, 3433 (1974); M. Chandrasekhar, J. B. Renucci, and M. Cardona, ibid. 17, 1623 (1978); H. Nakata, K. Yamada, and T. Ohyama, ibid. 60, 13269 (1999).

${ }^{7}$ K.-j. Jin, S. H. Pan, and G. Z. Yang, Phys. Rev. B 50, 8584 (1994); 51, 9764 (1995); S. H. Pan, Z. H. Chen, K.-j. Jin, J. Lu, T.-n. Zhao, Y. Huang, and G.-z. Yang, Z. Phys. B: Condens. Matter 101, 587 (1996).

${ }^{8}$ J. J. Hopfield, P. J. Dean, and D. G. Thomas, Phys. Rev. 158, 748 (1967).

${ }^{9}$ S. J. Xu, S.-J. Xiong, J. Liu, and H. Z. Zheng, Europhys. Lett. 74, 875 (2006).

${ }^{10}$ K.-j. Jin, J. D. Zhang, Z. H. Chen, G. Z. Yang, Z. H. Chen, X. H. Shi, and X. C. Shen, Phys. Rev. B 64, 205203 (2001).

${ }^{11}$ C. H. Henry and J. J. Hopfield, Phys. Rev. B 6, 2233 (1972).

${ }^{12}$ S. J. Xu, W. Liu, and M. F. Li, Appl. Phys. Lett. 77, 3376 (2000).

${ }^{13}$ S. J. Xu, S.-J. Xiong, and S. L. Shi, J. Chem. Phys. 123, 221105 (2005).

${ }^{14}$ C. B. Duke and G. D. Mahan, Phys. Rev. 139, A1965 (1965).

${ }^{15}$ B. Segall and G. D. Mahan, Phys. Rev. 171, 935 (1968).

${ }^{16}$ B. K. Meyer, Phys. Status Solidi B 241, 231 (2004).

${ }^{17}$ K. Huang and A. Rhys, Proc. R. Soc. London, Ser. A 204, 406 (1950).

${ }^{18}$ V. M. Fomin, V. N. Gladilin, J. T. Devreese, E. P. Pokatilov, S. N. Balaban, and S. N. Klimin, Phys. Rev. B 57, 2415 (1998); O. Verzelen, R. Ferreira, and G. Bastard, Phys. Rev. Lett. 88, 146803 (2002). 
Applied Physics Letters is copyrighted by the American Institute of Physics (AIP). Redistribution of journal material is subject to the AIP online journal license and/or AIP copyright. For more information, see http://ojps.aip.org/aplo/aplcr.jsp 